\title{
Interconnection of Distributed Energy Resources
}

\section{Emerson Reiter}

April 19, 2017

NREL/PR-7A40-68595 


\section{Goals for this Session}

- Understand the relationships between different aspects of interconnection

- Discuss best practices and lessons from US states and utility territories

- Provide an update on technical advances and standards for interconnection 
Motivations 


\section{Solar Energy: Cost}

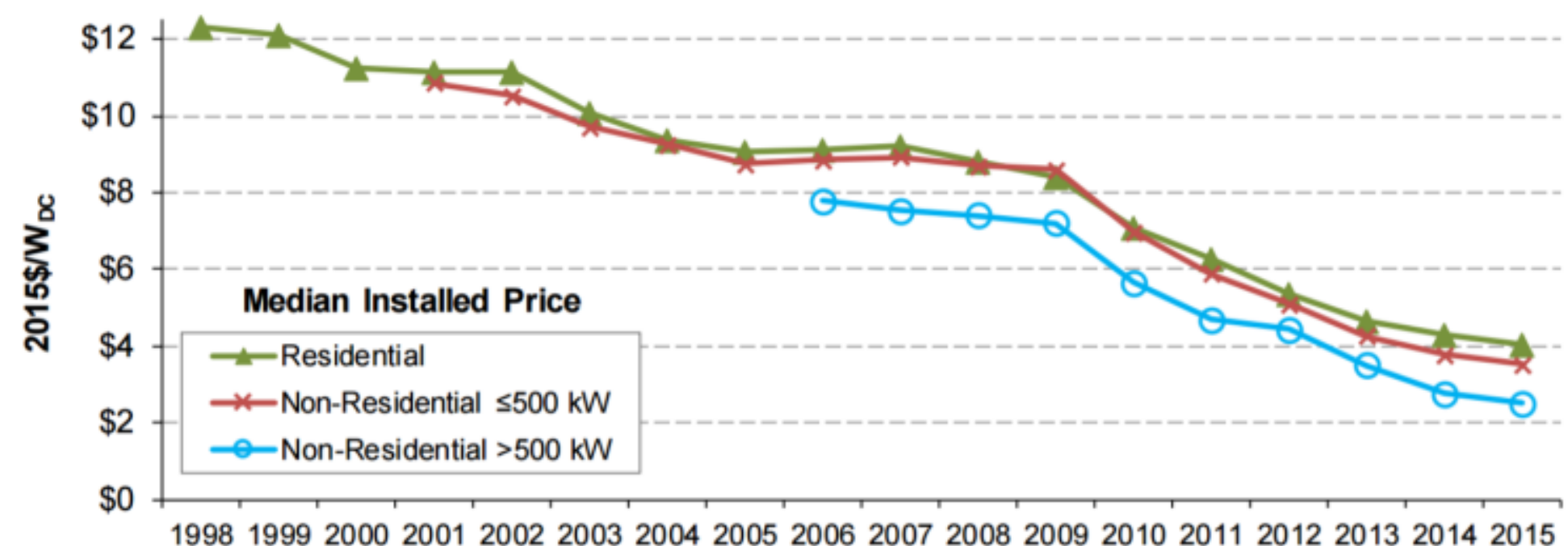

Installation Year

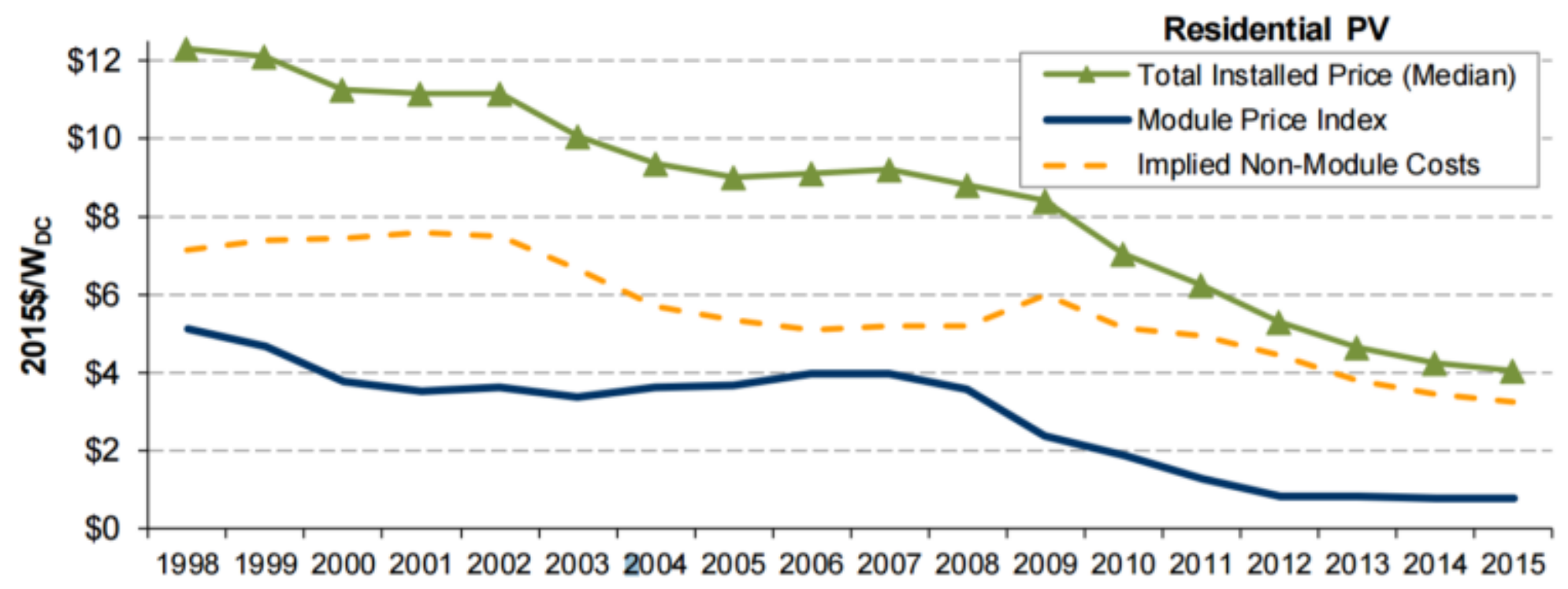




\section{Solar Energy: Resources}

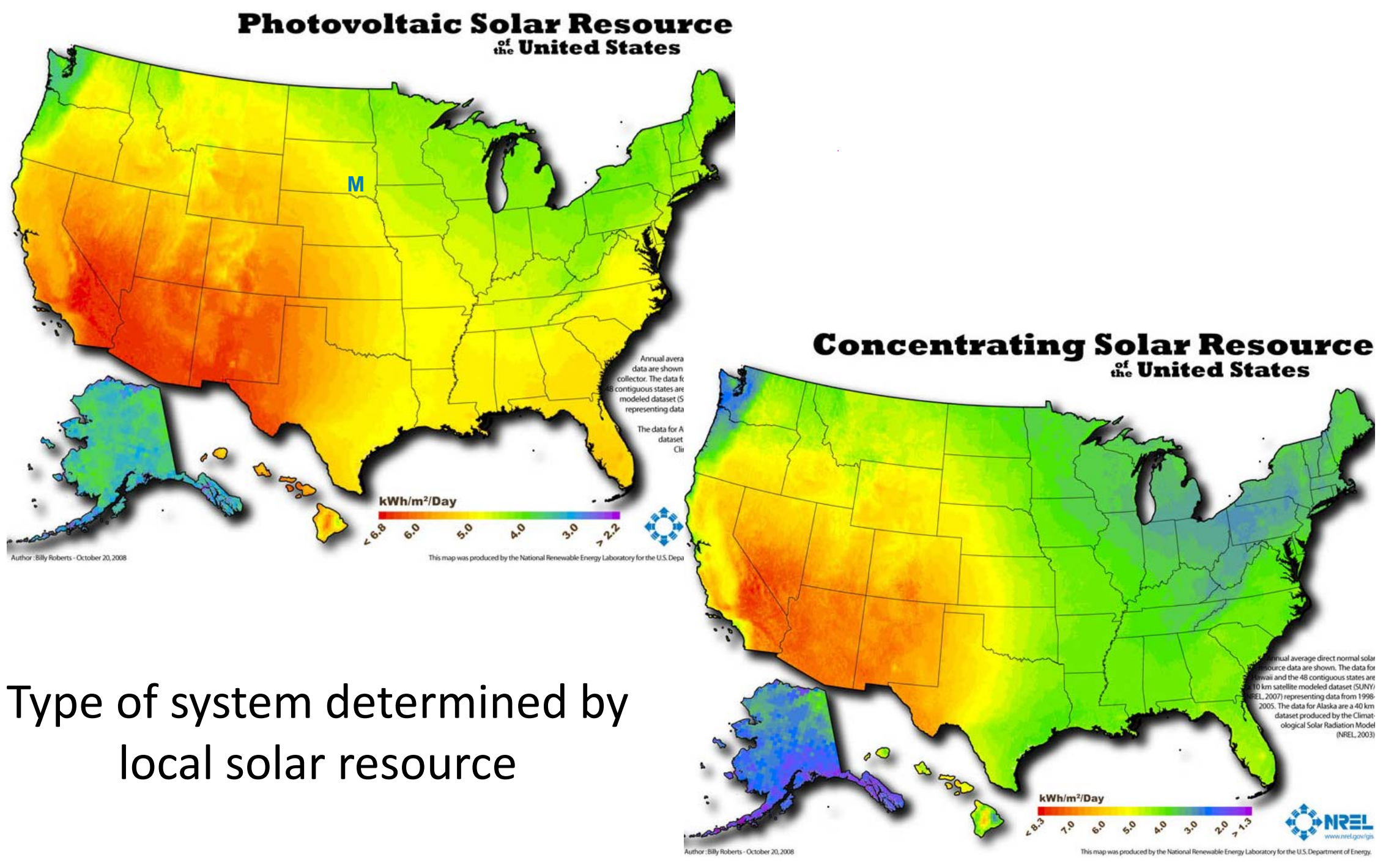




\section{Solar Energy: Utility Experiences of 10x Growth}

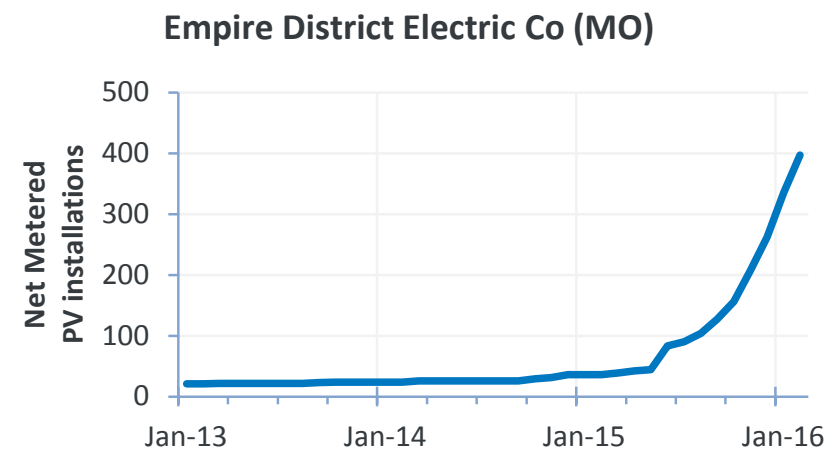

Modesto Irrigation District (CA)

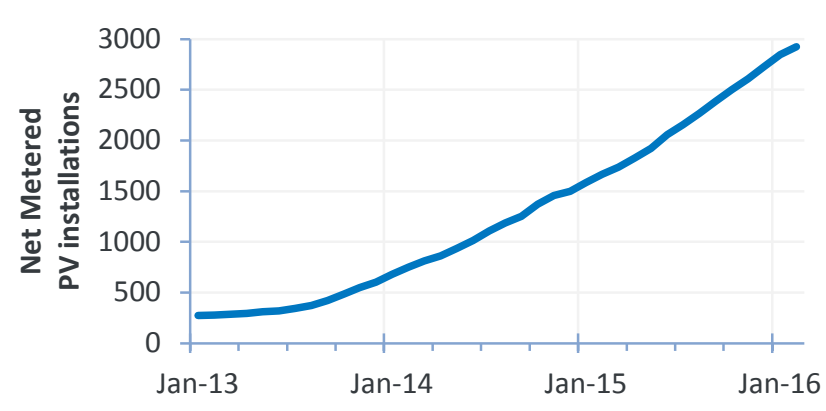

Nevada Power Co (NV)

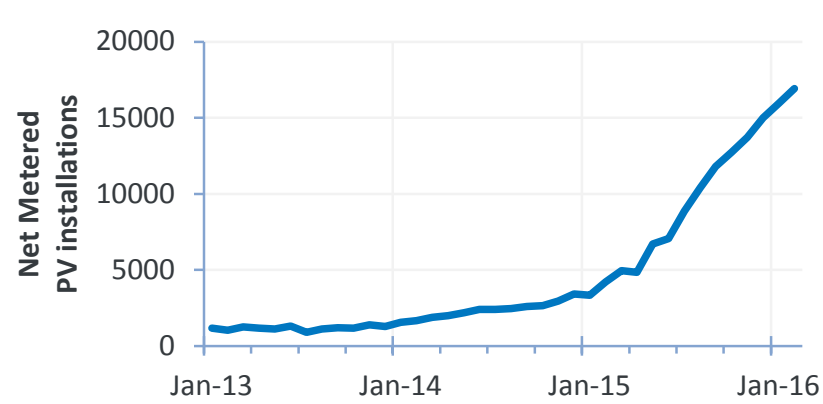

Story: Strong rebates, implemented in May 2015, push solar into wide viability.

Story: Growing economic viability overcomes decline in state incentives to drive steady growth.

Story: Solar reaches economic "tipping point" in late 2014, attracting scale from national installers. Pre-Dec 2015 installs were granted grandfathering. 


\section{Solar Energy: TVPPA Context}

\section{Utility Name}

Nashville Electric Service

Johnson City - (TN)

Southwest Tennessee E M C

Joe Wheeler Elec Member Corp

Gibson Electric Members Corp

Fort Loudoun Electric Coop

Decatur Utilities

City of Newport

Tennessee Valley Electric Coop

Tri-State Electric Member Corp

City of Rockwood - (TN)

City of Hopkinsville

Bolivar Energy Authority

Forked Deer Electric Coop, Inc

McMinnville Electric System

Guntersville Electric Board
Total

Customers

378,117

77,025

49,589

42,812

34,436

31,571

26,492

21,497

19,442

18,475

14,566

12,874

11,058

9,871

8,002

6,331
Additional DG systems/mo for 10x growth over 3 yrs 


\section{Intro to the Interconnection Process}




\section{Who does what?}

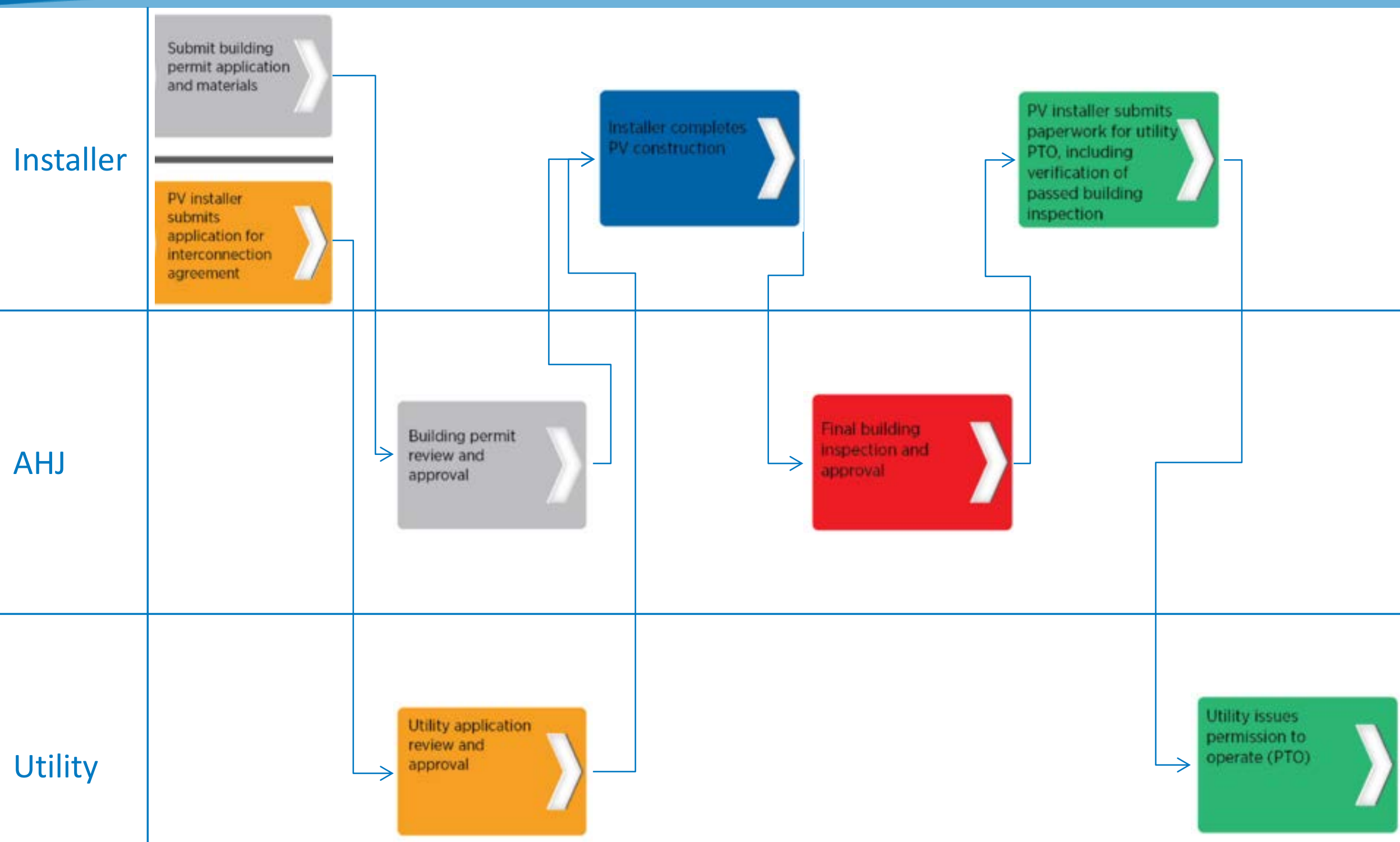




\section{What are the utility's typical responsibilities?}

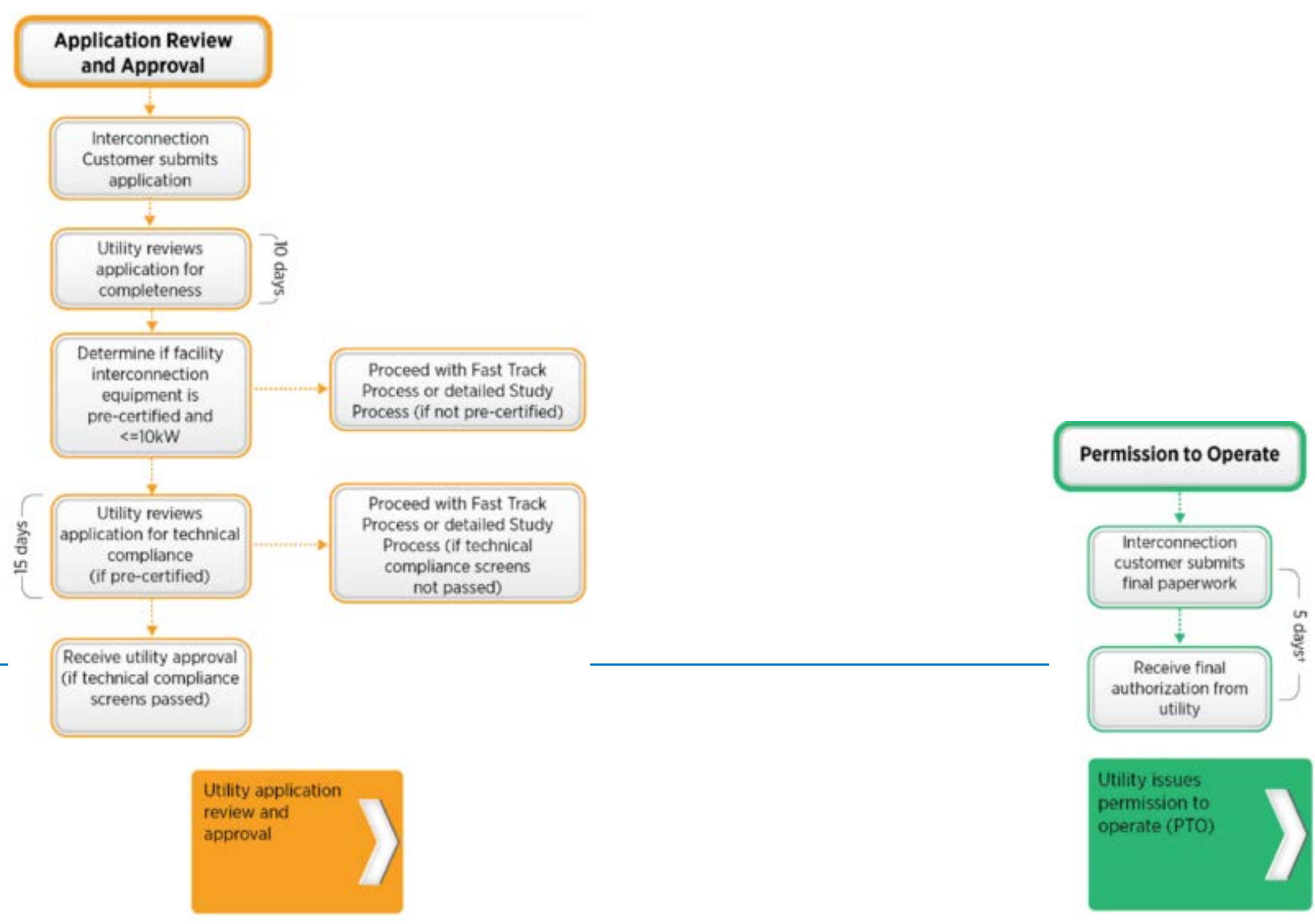




\section{Standards, Codes, and}

\section{Interconnection Requirements}




\section{Standards \& Codes are Foundational}

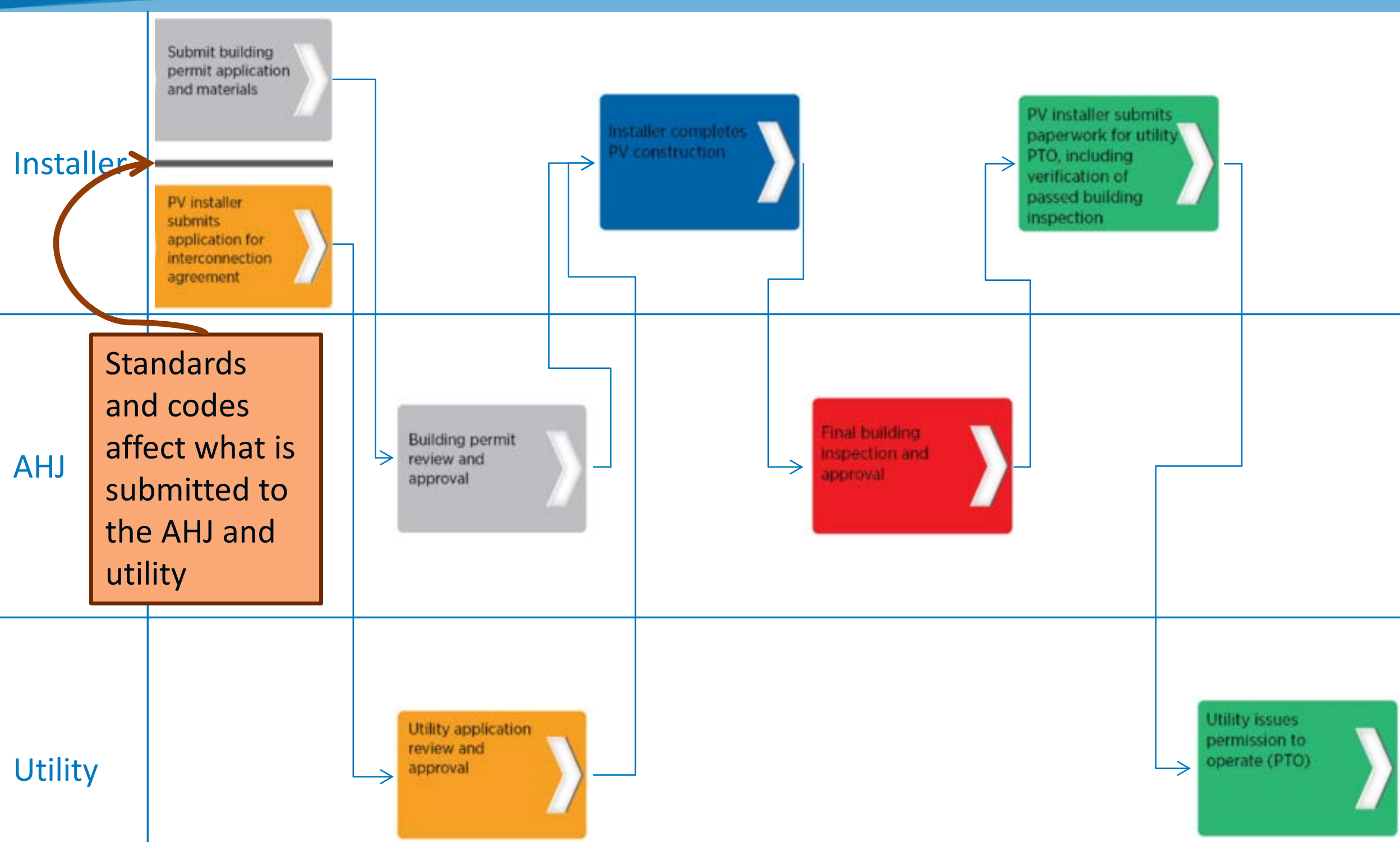




\section{Interconnection Codes \& Standards}

Electric Utility T\&D Systems

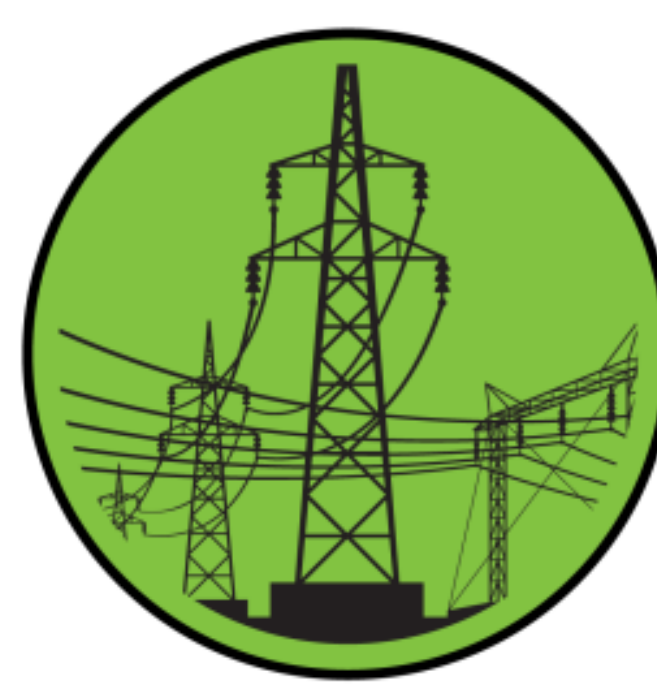

National Electrical Safety Code (NESC) and Utility Manual of Safe Practices

ANSI C84.1
PCC (Point of Common Coupling)
Industrial, Commercial, Residential Buildings

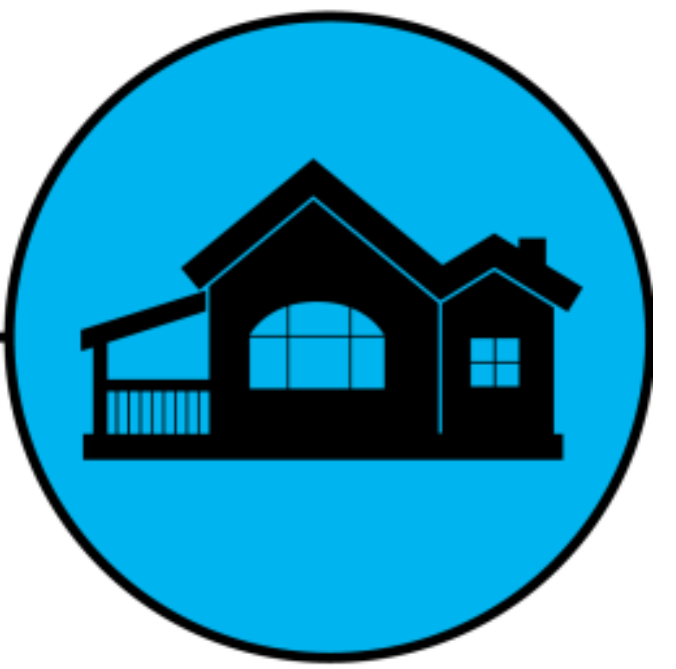

National Electrical Code (NEC)

IEEE 1547 \&

UL 1741

Family of Stds

UL 1703 
- Service Voltage - voltage at the point of delivery

- Range A (114-126V) is favorable, Range B (110-127V) is tolerable

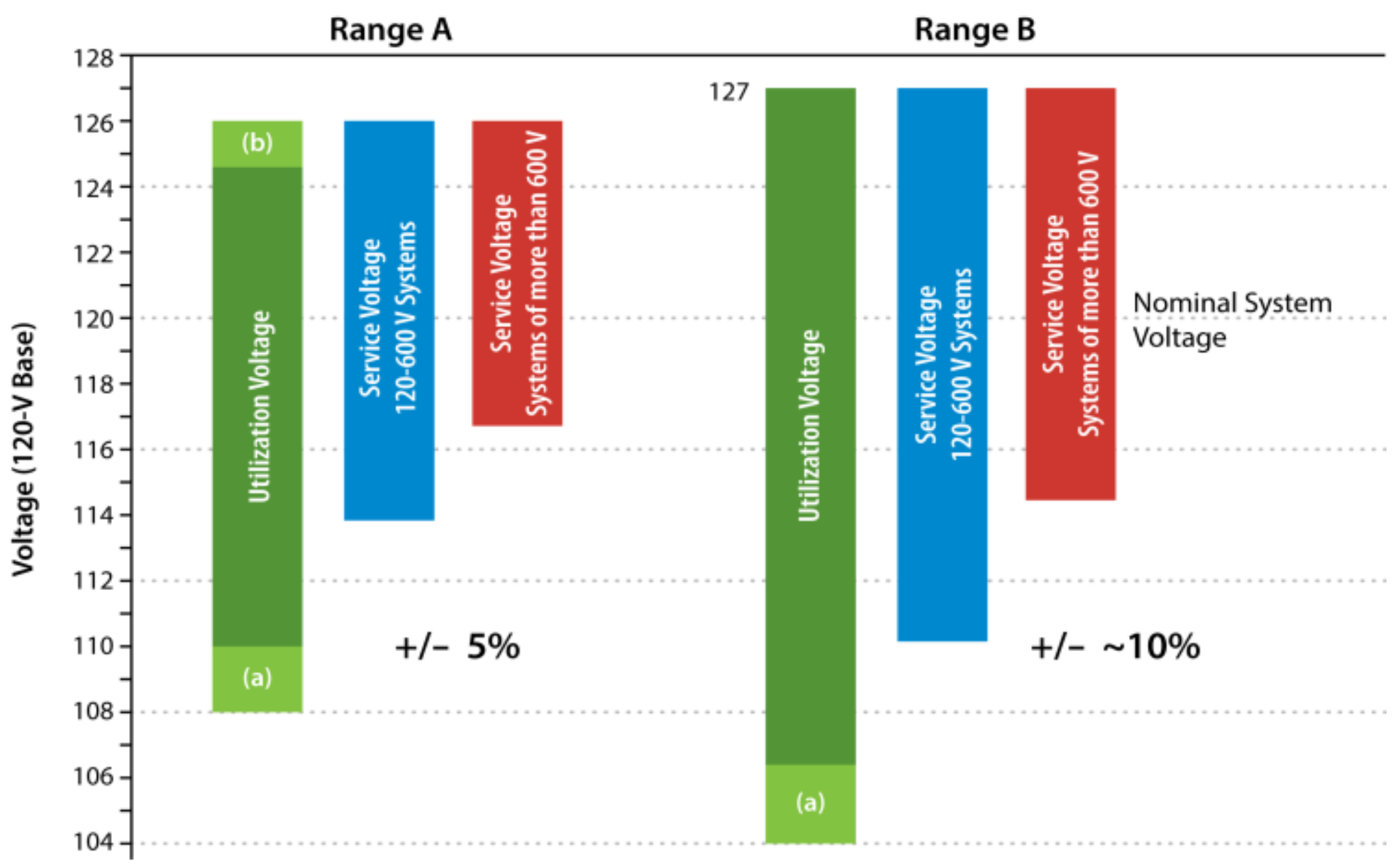




\section{IEEE $1547^{\mathrm{TM}}$ (under full review/revision)}

\section{- Provides a uniform standard for interconnection of DR with EPS}

- Set of standards govern performance, operation, testing, and safety

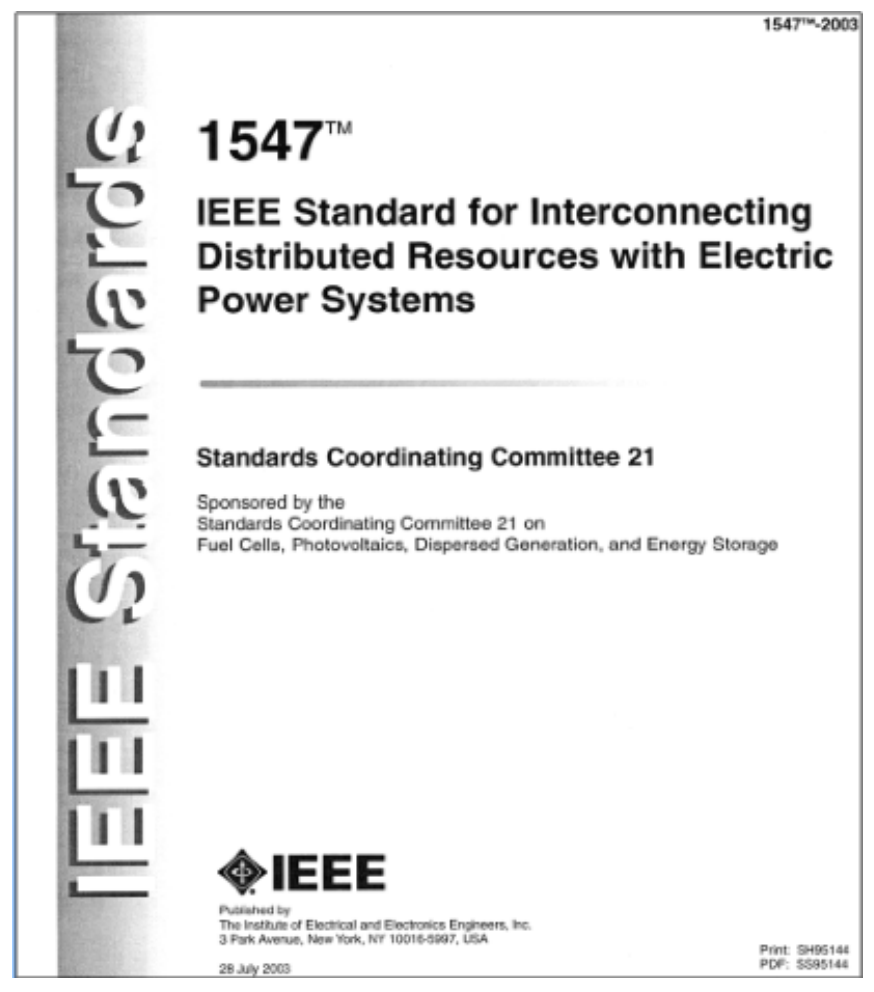

IEEE Std 1547.2 ${ }^{\text {m }}$ (2008) Application Guide for IEEE 1547 Standard for Interconnecting Distributed Resources with Electric Power Systems

IEEE Std 1547.3 $3^{\text {IM }}$ (2007) Guide for Monitoring Information Exchange, and Control of Distributed Resources with Electric Power Systems

IEEE Std $1547.4^{\mathrm{m} M}$ (2011) Guide for Design, Operation, and Integration of Distributed Resource Island Systems with Electric Power Systems

IEEE Std 1547.6 ${ }^{\mathrm{rm}}$ (2011) Recommended Practice for Interconnecting Distributed Resources with Electric Power Systems Distribution Secondary Networks

IEEE Std 1547.7 $7^{\text {TM }}$ (2013) Guide to Conducting Distribution Impact Studies for Distributed Resource Interconnection

IEEE Std P1547.8 ${ }^{\text {m }}$ Draft Recommended Practice for Establishing Methods and Procedures that Provide Supplemental Support for Implementation Strategies for Expanded Use of IEEE Std 1547-2003 


\section{National Electrical Code (NEC)}

\section{National Fire Protection Association (NFPA) 70}

- Adopted as law in most U.S. states, other areas

- Applies to Residential, Commercial, and Industrial facilities

- Often used in utility power plants, service centers

- Articles 690 (PV systems) \& 705 (Interconnected Electric Power Production Sources)

- New Article 691 in next code cycle (Utility-Scale PV) 
Inverters, Converters, Controllers, and Interconnection System Equipment for Use with Distributed Energy Resources

- Adopted as Legal Requirement in most U.S. states

- Applies to the inverter and interconnection equipment

- Inverters should be "listed" to this standard

- Harmonized with IEEE 1547

- Underwriters Laboratories - Standard for Safety

- UL 1741 has been updated for "Smart Inverter Use"

- UL 1741 Supplement A (SA) 


\section{Dependencies in Technical Standards}

\section{Equipment Safety}

Prior to Supplement A, UL 1741 did not have a testing procedure for advanced inverters

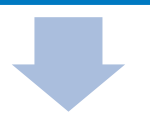

Without a developed testing program under UL 1741, advanced inverters could not achieve UL listing

Installations using advanced inverters which are not ULlisted cannot comply with the National Electrical Code

Installations which do not comply with the National Electrical Code will violate state or local building codes

\section{Interconnection Performance}

IEEE 1547-2003 does not allow inverters to perform "advanced" functions beyond those in IEEE 1547a-2014

In most states, an inverter which violates IEEE 1547-2003 does not meet state interconnection standards

Inverter deployments which do not meet state standards will not pass utility interconnection processes 
Technical Screens 


\section{Technical Screens are Key to Application Review}

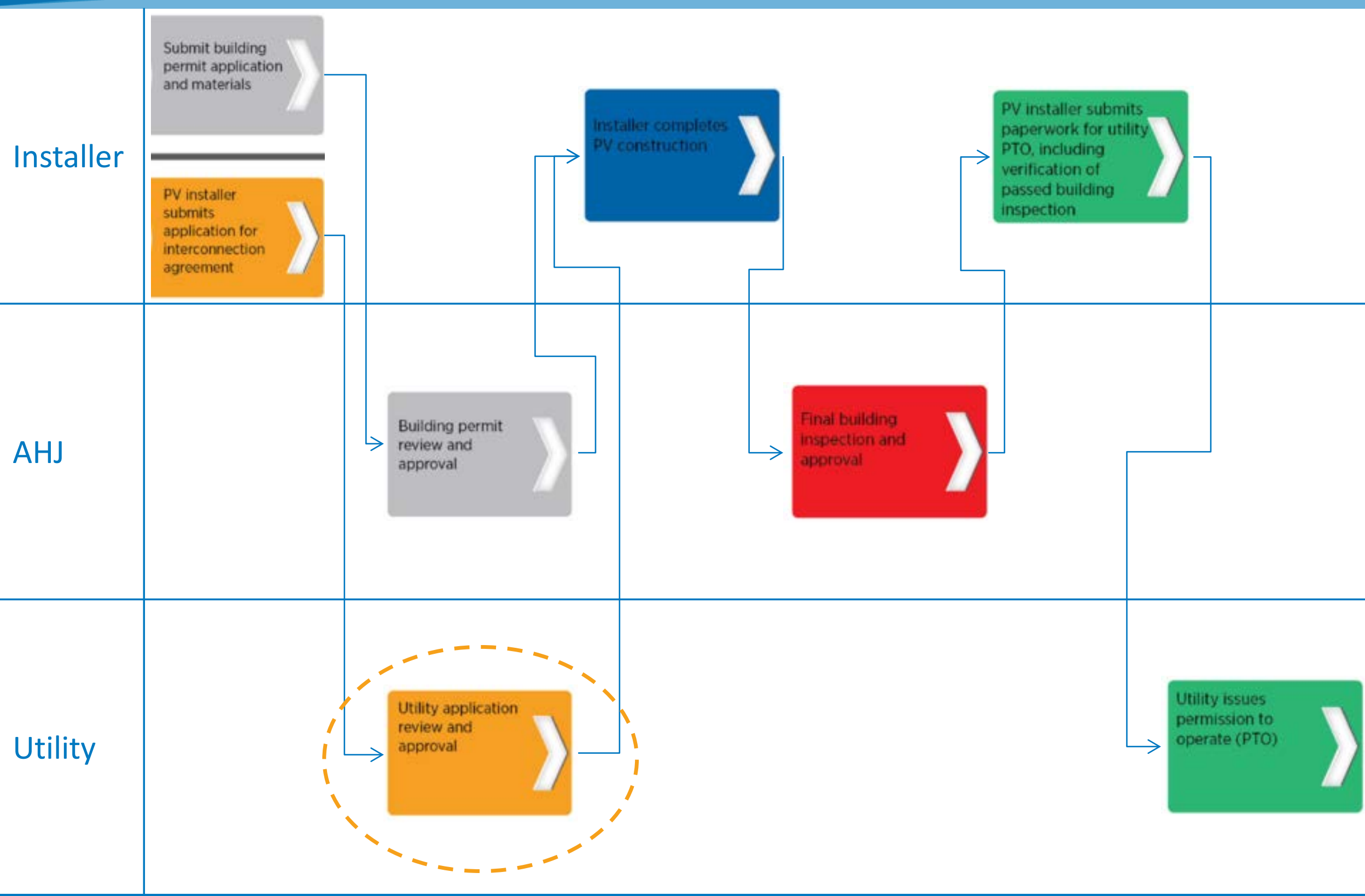




\section{Generic Utility Interconnection Review Process}

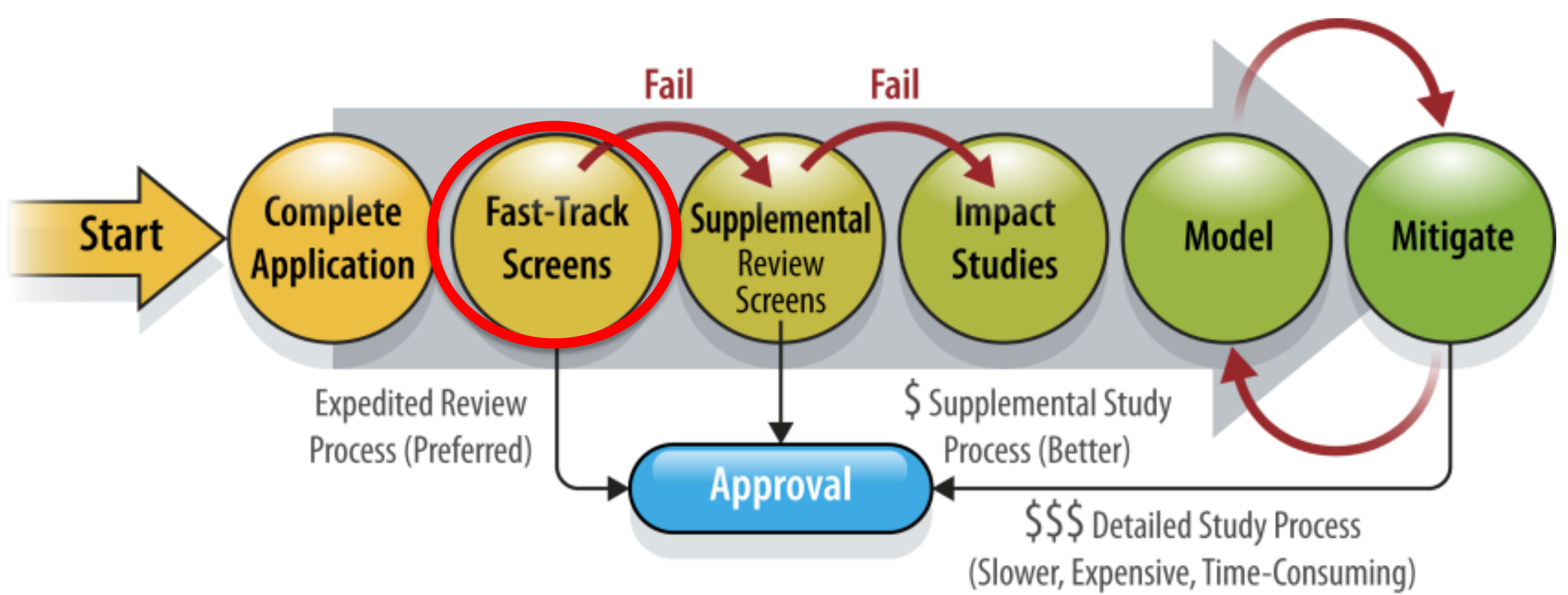




\section{Fast Track Screens (from FERC SGIP)}

1. Aggregated DG $<15 \%$ of peak load on line section (Penetration Screen)

2. For connection to a spot network: DG is inverter-based, aggregated DG capacity is $<5 \%$ of peak load $\&<50$ $\mathrm{kW}$

3. Aggregated DG contribution to maximum short circuit current is $<10 \%$

4. Aggregated DG does not cause protective device to exceed $87.5 \%$ of short circuit interrupting capability

5. DG interface is compatible with type of primary distribution line (Wye/Delta)
6. For a single-phase shared secondary, aggregated DG capacity $<20 \mathrm{~kW}$

7. Resulting imbalance $<20 \%$ of service transformer rating of 240 V service

8. Aggregated transmission connected DG capacity <10 MW for stability-limited area

9. Construction not required for interconnection 


\section{Supplemental Review May Occur if Fast-Track not Achieved}

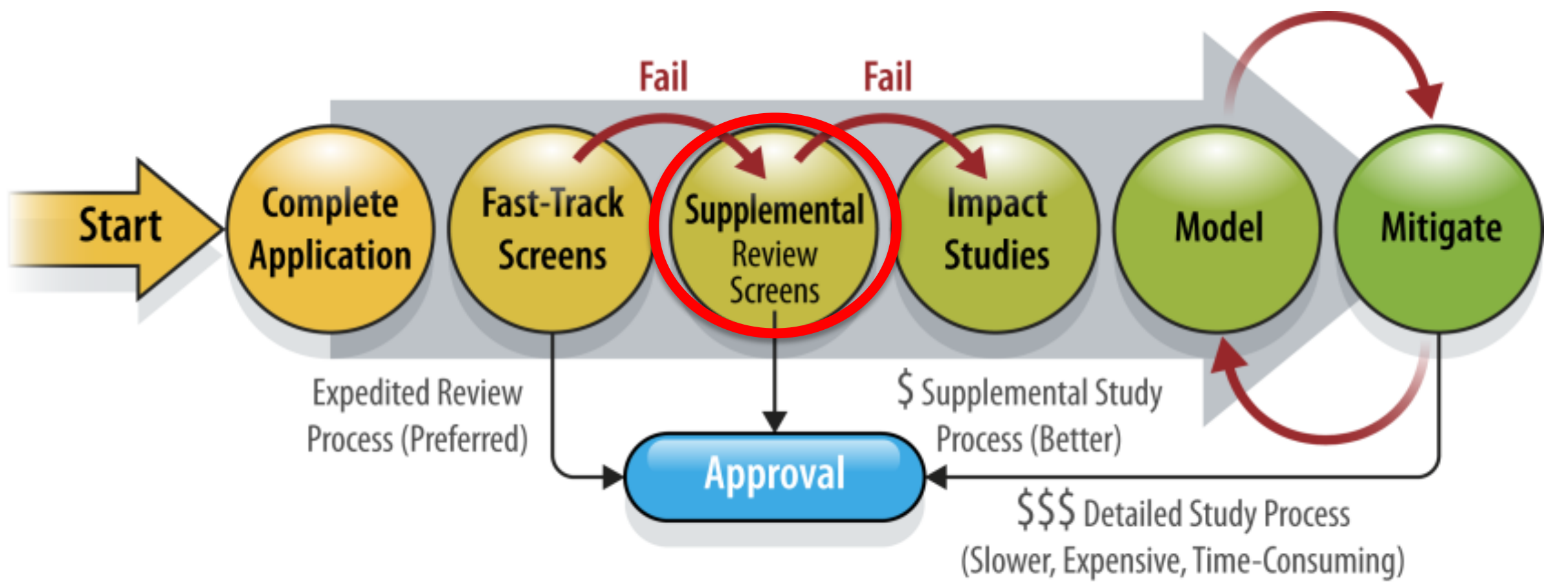




\section{Supplemental Screen Examples}

- Evaluate transformer serving customer (Is it sufficient, can it be easily changed)

- Evaluate secondary lines serving customer

- Consider location of proposed PV to substation (short distance, large conductors, etc.)

- If Penetration Screen failed, consider other metrics such as $100 \%$ of minimum daytime load on line section (recommended by FERC) 


\section{Tension in Screening Process}
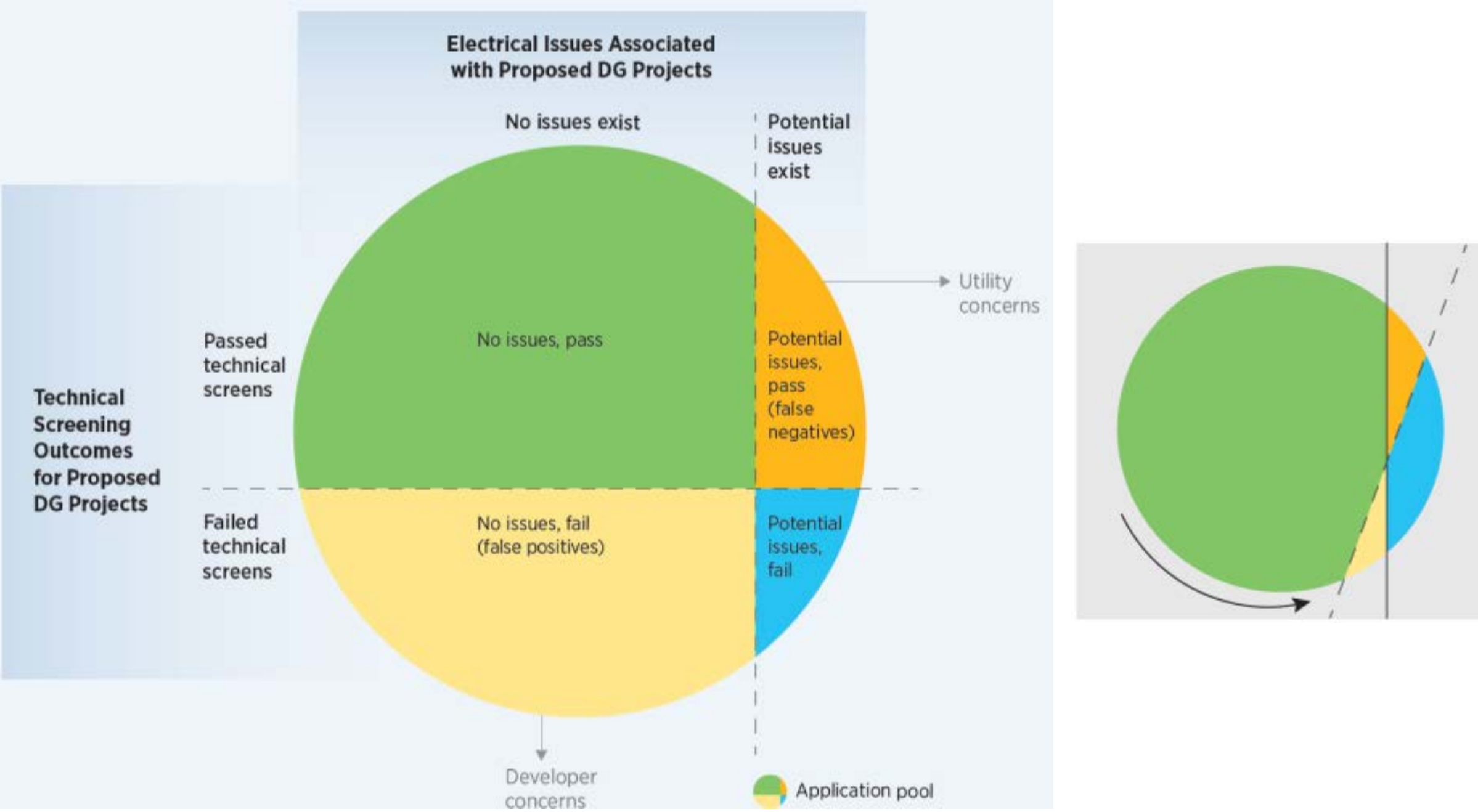


\section{Interconnection Studies and Mitigations}




\section{Detailed Studies Can Occur in Application Review}

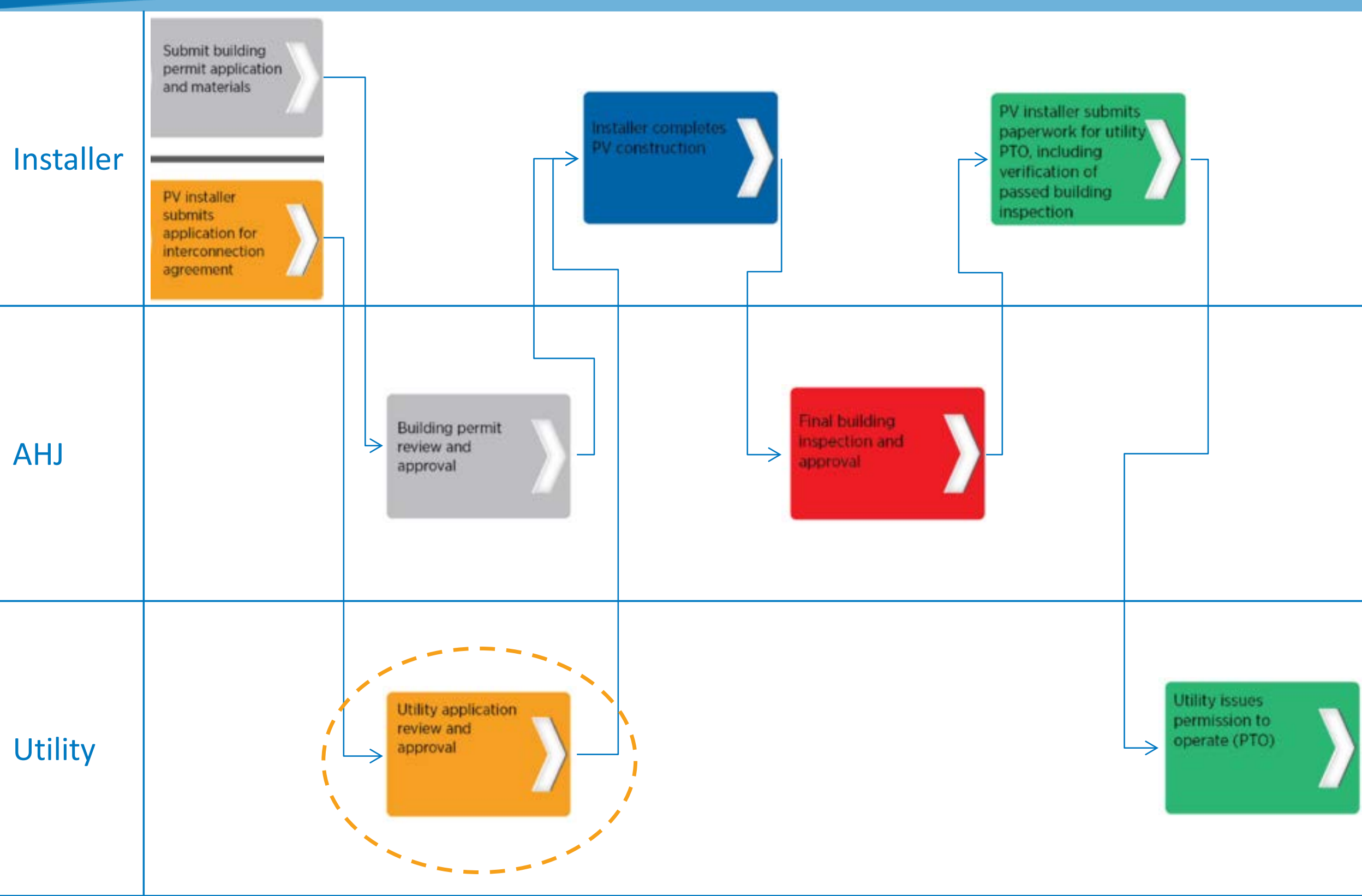




\section{Impact Studies Are Triggered if Screens are Failed}

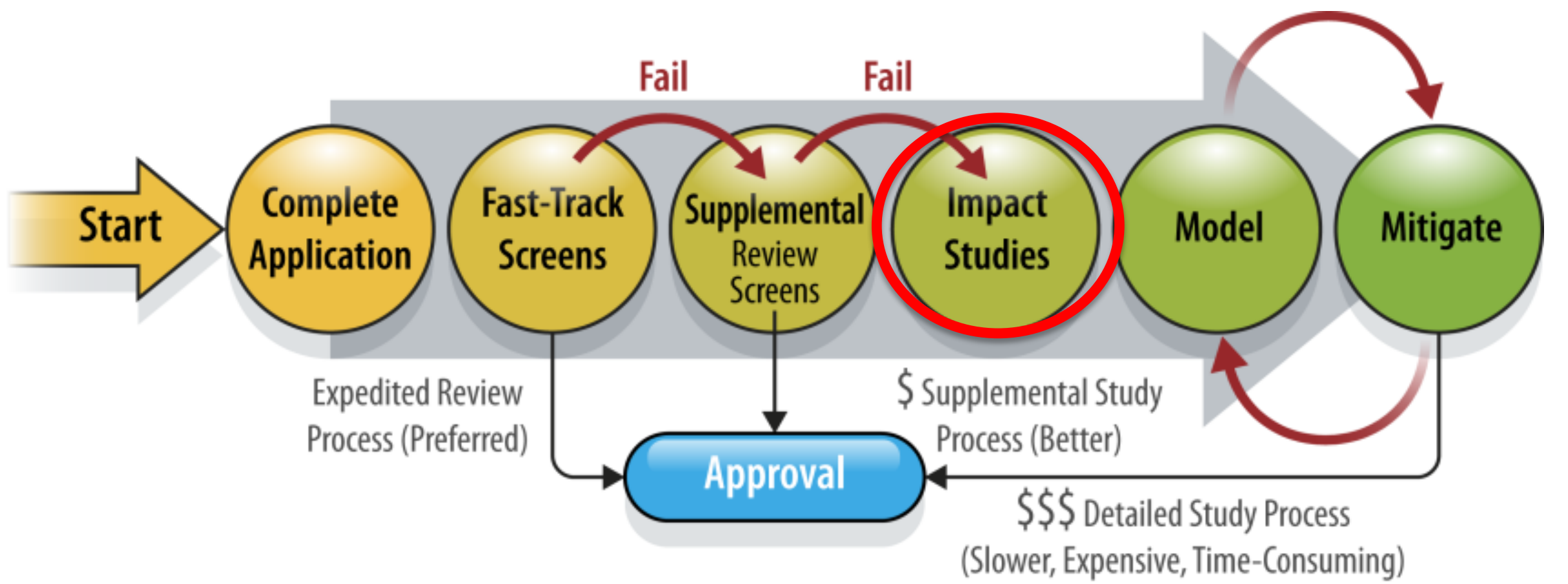




\section{Utility Survey (SGIP Focused)}

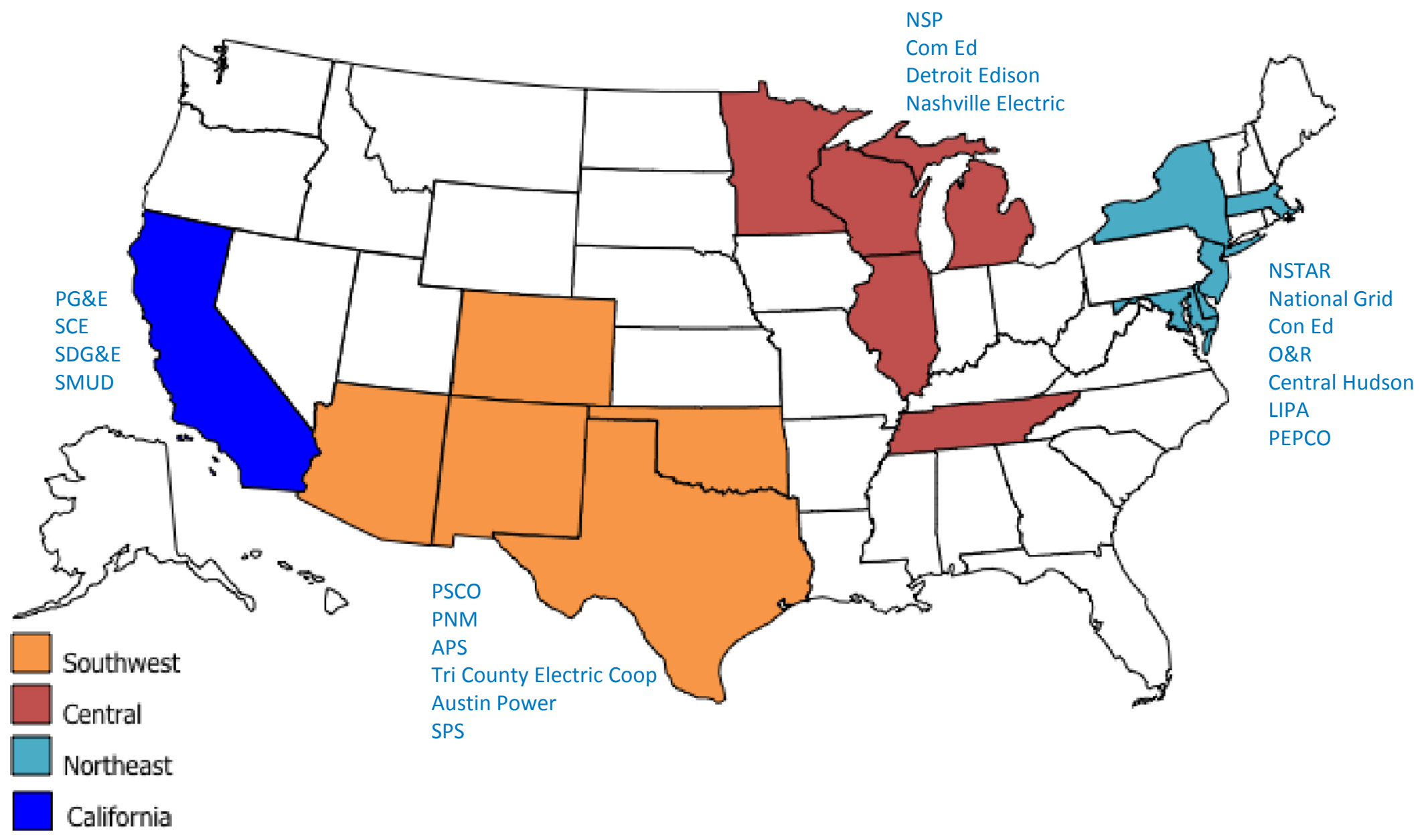




\section{Major Utility Concerns}

Voltage Regulation $76 \%$

Reverse power flow 52\%

Protection system coordination $48 \%$

Increased duty of line regulation equipment 38\%

Unintentional islanding 38\%

Secondary network protection $29 \%$

Variability due to clouds $24 \%$

Increased switching of capacitors 19\% 


\section{Voltage Regulation - No PV}
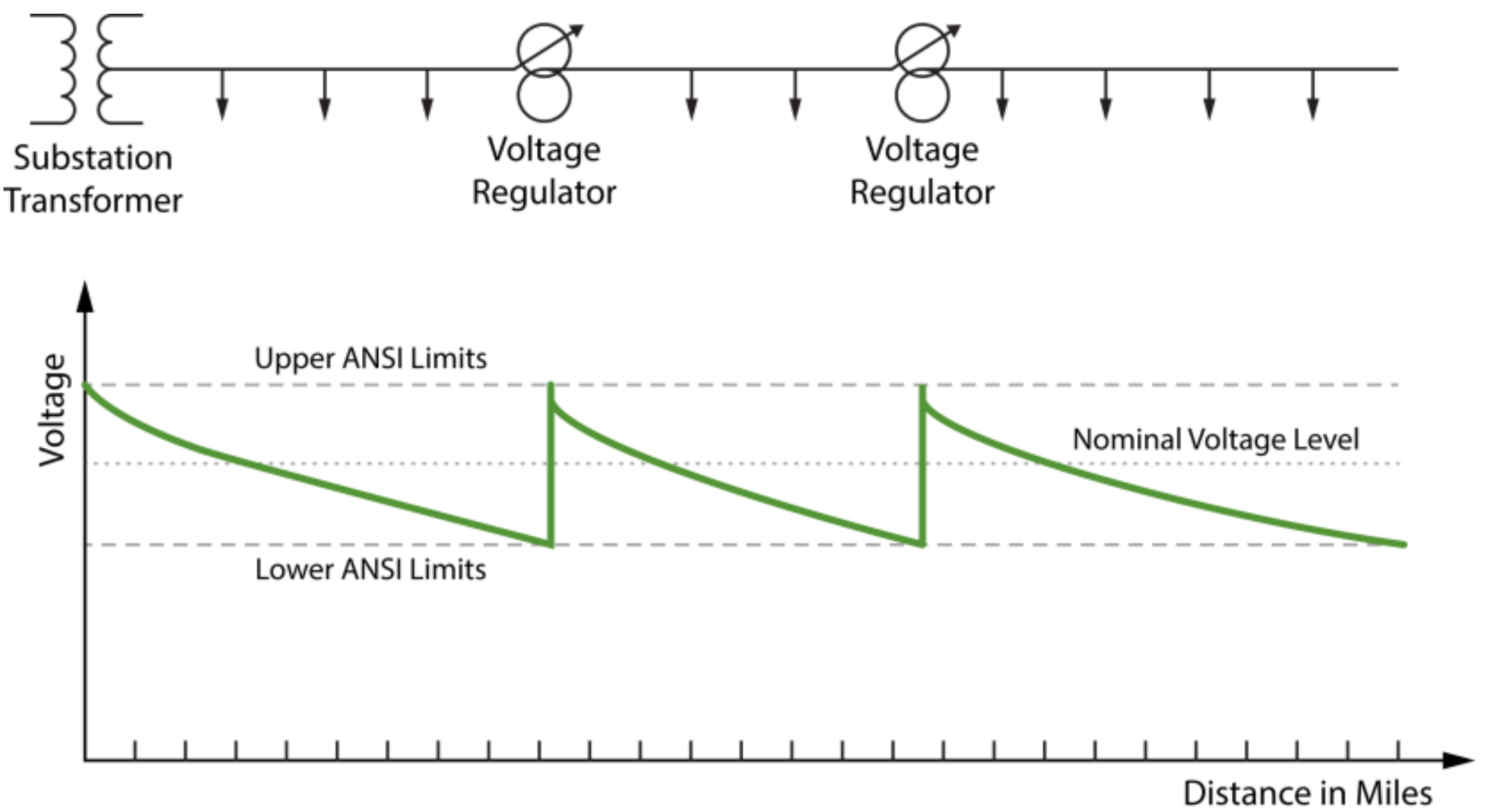


\section{Voltage Regulation - w/ PV}
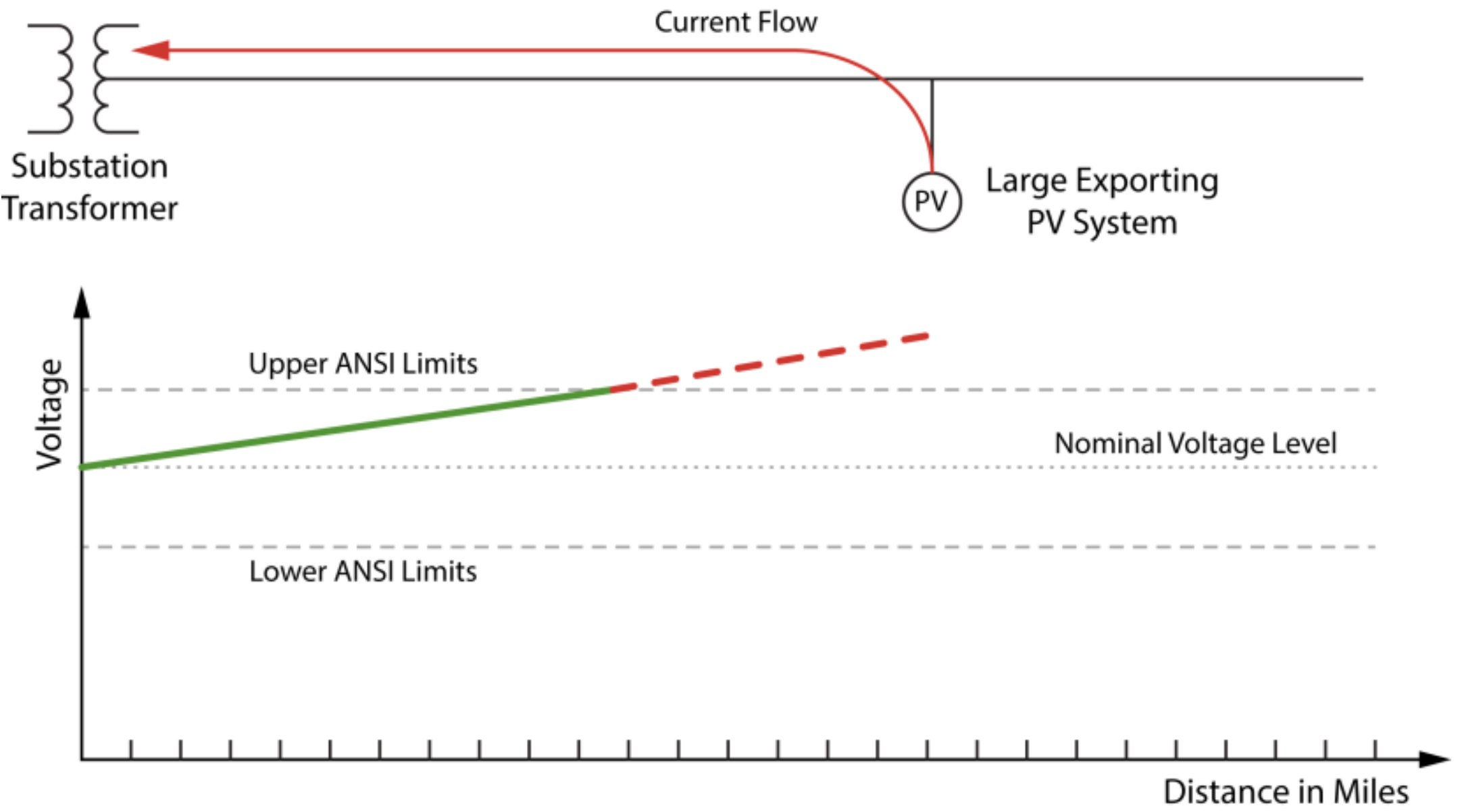


\section{Voltage Regulation - w/ PV}
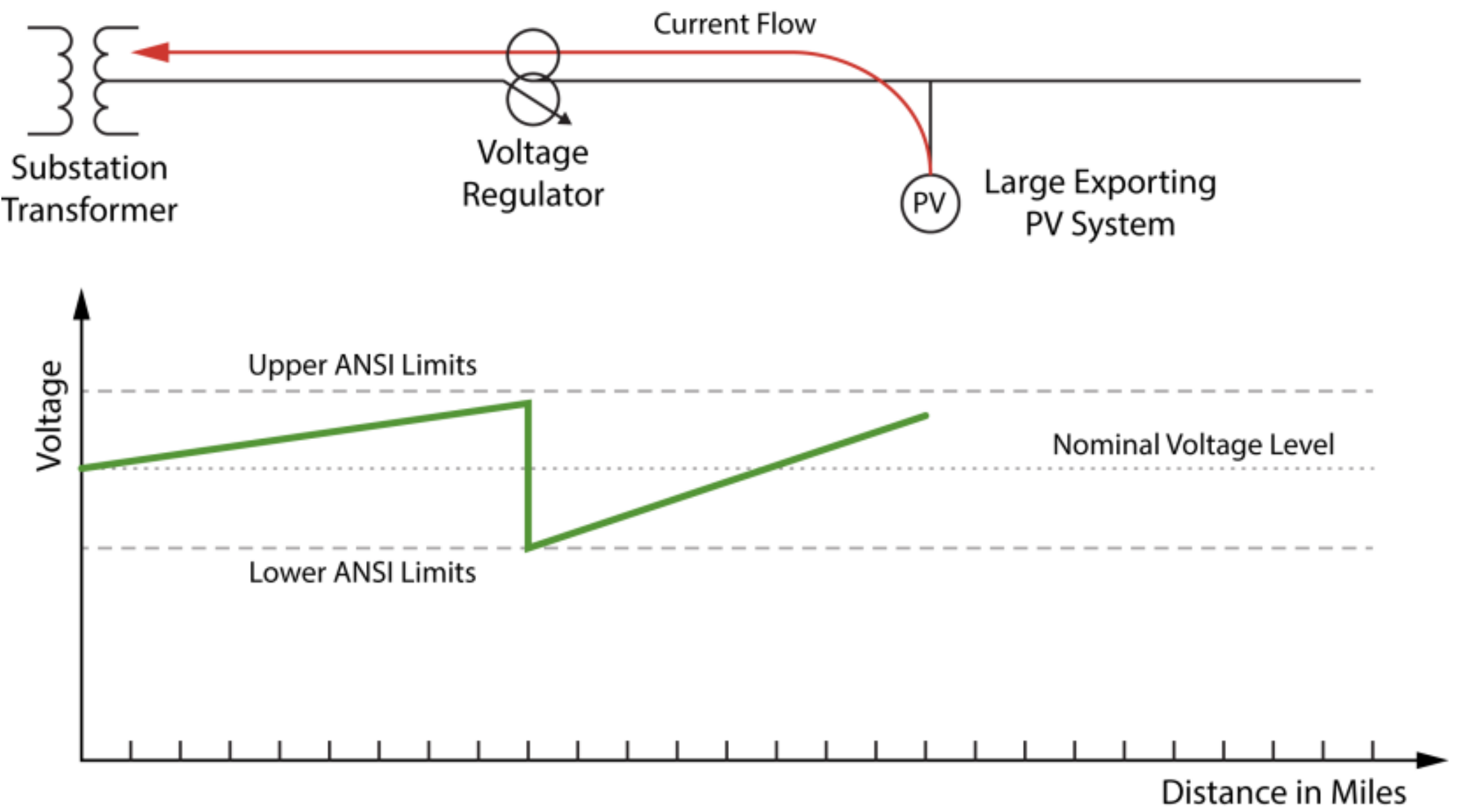


\section{Detailed Impact Study Types}

Most utilities employ one or more of the following;

- Power Flow (common)

- Short Circuit (common)

- Voltage (common)

- Feasibility

- Facility

Most Commonly used

Distribution Software

- Flicker

- Power Quality

- Dynamic/Transient Stability

- Electromagnetic Transient

- SynerGi

- CymDist

- Milsoft Windmil

- ASPEN 


\section{Mitigation Tools Available}

Tools in the "Toolbox"

- Upgrade Line Section

- Modify Protection Scheme (breakers \& fuses)

- Voltage Regulation Devices (Add or chance control)

- Direct Transfer Trip

- Advanced Inverters Required

- Communication \& Control Technologies

- Power Factor Controls (Often advanced inverters)

- Grounding Transformers

- Static VAR Compensators

- Capacitor Control Modifications

- Volt/VAR Controls 


\section{Interconnection as Business Process}


How Can a Utility Improve Customer Service?

1. Be clear

2. Be quick 


\section{Be Clear}

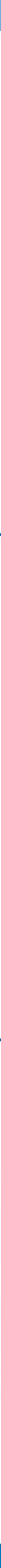




\section{How Can a Utility Improve Customer Service?}

\section{Be clear}

- Easily accessible interconnection guidelines based on accepted standards

- Model procedures: FERC Small Generator Interconnection Procedure (SGIP), IREC

- Designated points of contact at utilities

- Ideally, a real person, not a generic inbox

- Single channel for submission of application materials (electronic)

- Eliminates coordination of mail, fax, e-mail applications

- Provide a way for installers to track progress and/or clear communication of decisions, timelines, and cost

- Email templates of standard updates 
2. Be Quick

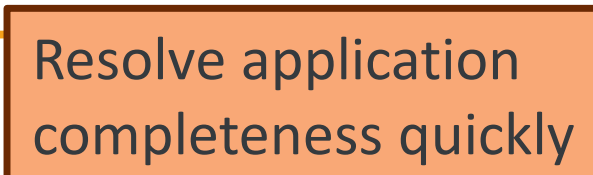

Use screens to speed processing, reduce burden on staff

ail

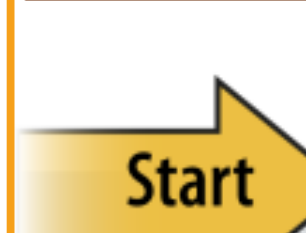

Application

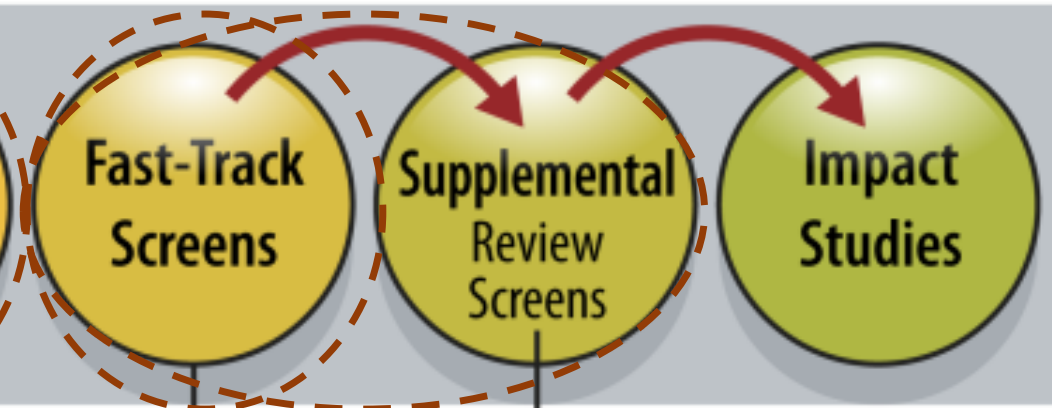

Expedited Review

Process (Preferred)

Consider fast-track process (e.g. inverterbased, under $10 \mathrm{~kW}$ )

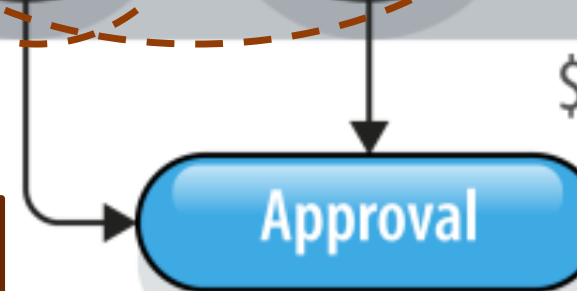

\$Supplemental Study

Model

Process (Better)

\section{$\$ \$ \$$ Detailed Study Process}

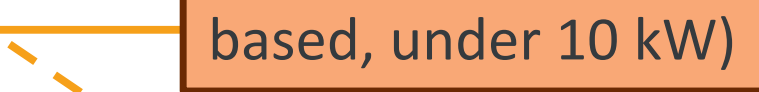

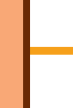

\section{(Slower, Expensive, Time-Consuming)}

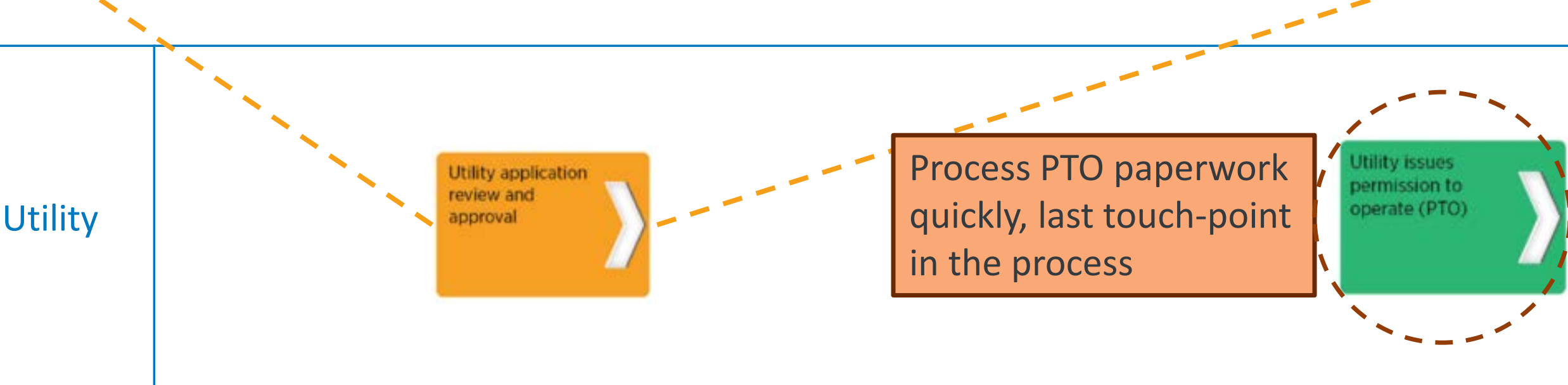




\section{How Can a Utility Improve Customer Service?}

\section{Be clear}

- Easily accessible interconnection guidelines based on accepted standards

- Designated points of contact at utilities

- Single channel for submission of application materials (electronic)

- Provide a way for installers to track progress and/or clear communication of decisions, timelines, and cost

\section{Be quick}

- Use checks for completeness

- Engaging installers quickly can avoid long, frustrating waits

- Fast-track process (e.g. inverter-based systems under $10 \mathrm{~kW}$ )

- Included in FERC SGIP and IREC model procedures

- Use screens to analyze systems quickly, reduce analytical burden on staff

- Move paperwork quickly and efficiently

- Permission to Operate is simply confirming documents received and in order, last touchpoint with customer in the process

- Bonus points: coordinate with local permitting process 


\section{Reducing Time and Cost of Interconnection}

Municipal utilities may have unique opportunities to streamline the interconnection, incentive and building permit approval process as a result of having one centralized authority in control of the processes 


\section{Municipalities Can Merge Permitting and Interconnection}

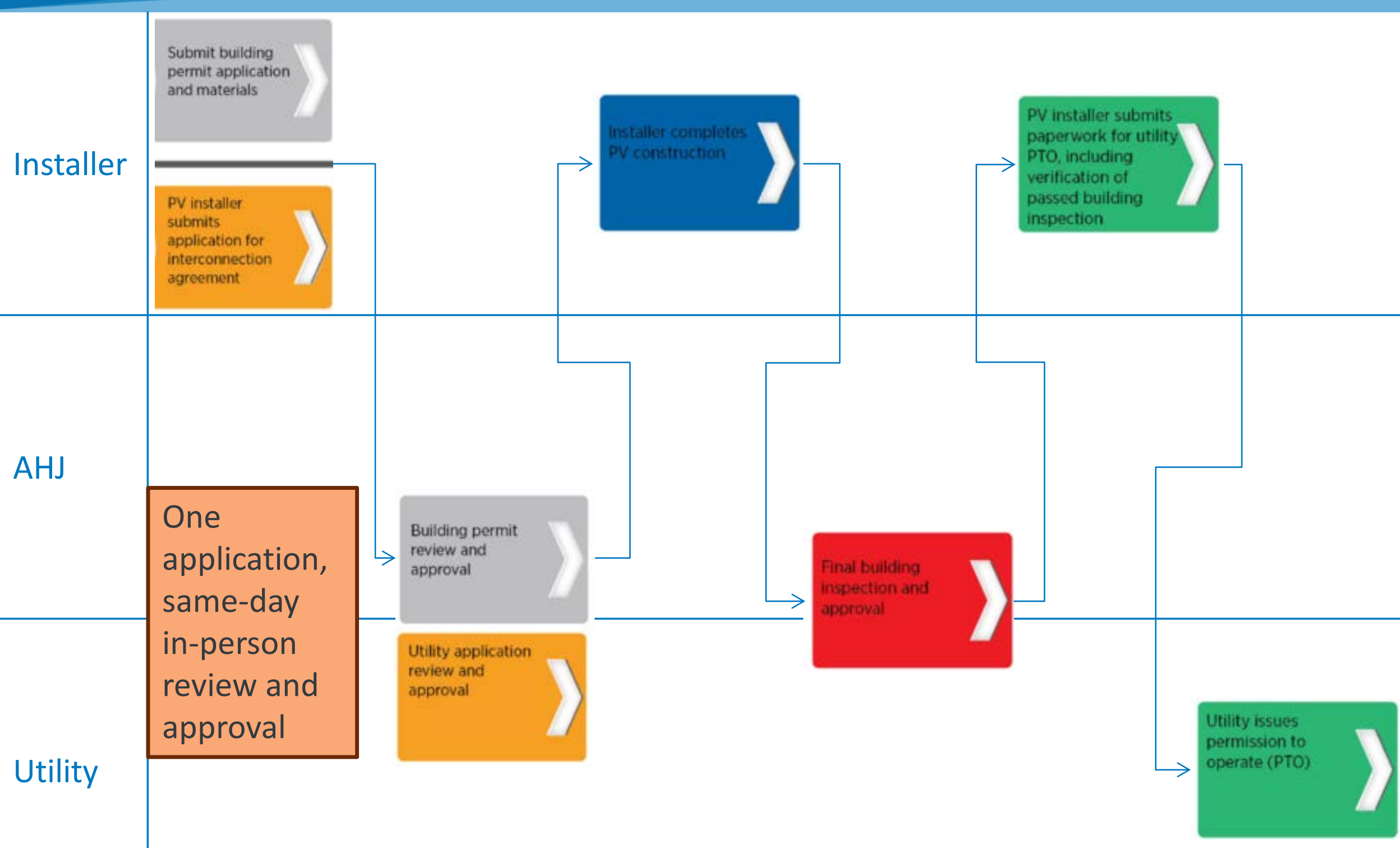




\section{Advanced or "Smart" Inverters}




\section{What is a "Smart" Inverter - Functions}

\section{Multiple efforts have been made to standardized function set for smart inverters}

\section{Key capabilities include (but not limited to):}

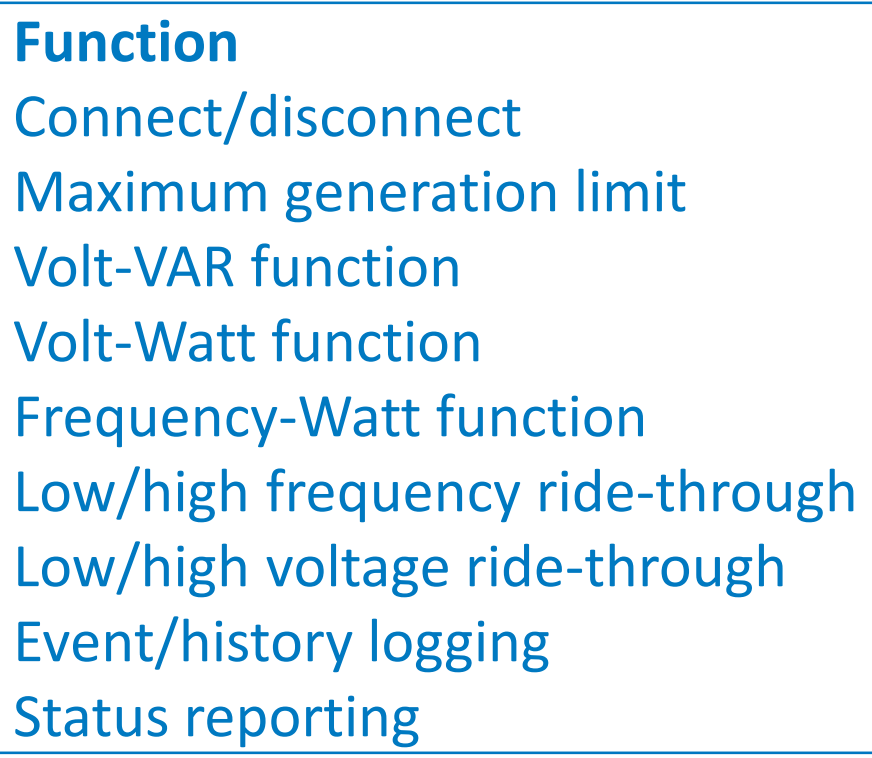

\section{Description}

Electrically connects to or disconnects from the grid Constrains real power output Adjusts reactive power output based on service voltage Adjusts real power output based on service voltage Adjusts real power output based on service frequency Defines frequency range for which inverter remains on-line Defines voltage range for which inverter remains on-line Log and report standardized set of events Report current device status 


\section{What is a "Smart" Inverter - Benefits}

- Increased grid stability

- Local - voltage control from volt-VAR and volt-Watt functions

- System - greater resiliency during frequency deviations

- Increased visibility into distribution system conditions via data collection

- Potential to defer investments in other distribution system hardware 


\section{Key Barrier - Technical Standards}

Key Standards for Advanced Inverters

- UL 1741

- Developed by Underwriter's Laboratory (UL)

- Primarily an equipment safety standard

- Sets design requirements and testing procedures

- IEEE 1547

- Developed by Institute of Electrical and Electronics Engineers (IEEE)

- Primarily an interconnection performance standard

- Governs how inverters affect electrical conditions at the point of common coupling (PCC) with the grid 


\section{Key Barriers - Technical Standards}

\section{Standards acted as a barrier because other regulations rely on these measures}

\section{Equipment Safety}

Prior to Supplement A, UL 1741 did not have a testing procedure for advanced inverters

Without a developed testing program under UL 1741, advanced inverters could not achieve UL listing

Installations using advanced inverters which are not UL-listed cannot comply with the National Electrical Code

Installations which do not comply with the National Electrical Code will violate state or local building codes

\section{Interconnection Performance}

IEEE 1547-2003 does not allow inverters to perform "advanced" functions beyond those in IEEE 1547a-2014

In most states, an inverter which violates IEEE 1547-2003 does not meet state interconnection standards

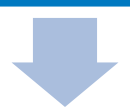

Inverter deployments which do not meet state standards will not pass utility interconnection processes 


\section{UL 1741 Supplement A (UL 1741 SA)}

Updated to include advanced functions

Approved September 2016

Several inverters have been certified to date

The UL 1741 SA consists of the following advanced inverter grid support utility interactive test plan:

\section{Required Tests (utilized by all SRDS)}

Anti-Islanding (with advanced features active during test)

Low/High Voltage Ride Through

Low/High Frequency Ride Through

Must Trip Test

Ramp Rate (Normal \& Soft-Start)

Specified Power Factor

Volt/Var Mode

Optional Tests (depends on SRD being utilized)

Frequency Watt

Volt Watt 


\section{IEEE 1547 full revision}

Complete overhaul of interconnection requirements

New content on:

- Reactive power

- Ride-through requirements

- DER on secondary networks

- Interoperability and communications

- Test and verification requirements

In balloting now, publication anticipated summer 2017 


\section{Key Barriers - Communications}

Communications bring challenges and benefits

Challenges

- Many layers of physical and software infrastructure

- Standardization to maximize value requires coordination across many parties

Benefits

- Enables certain high-value functions

- E.g. real power curtailment

- Supports more frequent updates to otherwiseautonomous functions

- Increases visibility for distribution management 


\section{Key Barriers - Communications}

\begin{tabular}{|c|c|c|c|c|}
\hline \multicolumn{2}{|c|}{ Communications Level } & \multirow{2}{*}{$\begin{array}{l}\text { Inverter Functions } \\
\text { Low- / High-voltage ridethrough }\end{array}$} & \multicolumn{2}{|c|}{$\begin{array}{l}\text { Proceedings and } \\
\text { Standards }\end{array}$} \\
\hline \multirow{11}{*}{ Autonomous } & \multirow{11}{*}{$\begin{array}{l}\text { - No communications } \\
\text { architecture needed } \\
\text { - Behavior controlled by } \\
\text { operating parameters } \\
\text { - Parameters defined at } \\
\text { system commissioning } \\
\text { - Parameters can be } \\
\text { adjusted, behavior } \\
\text { activated or deactivated } \\
\text { at later date via remote } \\
\text { or on-site changes }\end{array}$} & & \multirow{7}{*}{$\begin{array}{l}\text { SIWG } \\
\text { Phase } 1\end{array}$} & \multirow{3}{*}{$\begin{array}{c}\text { IEEE } \\
1547 \mathrm{a} \\
2014 \\
\end{array}$} \\
\hline & & Low- / High-frequency ridethrough & & \\
\hline & & Volt-var control & & \\
\hline & & Anti-islanding & & \\
\hline & & Ramp-rate controls & & \\
\hline & & $\begin{array}{l}\text { Provide reactive power } \\
\text { (via fixed power factor) }\end{array}$ & & \\
\hline & & Reconnect via "soft-start" & & \\
\hline & & Frequency-watt & \multirow{4}{*}{$\begin{array}{l}\text { SIWG } \\
\text { Phase } 3\end{array}$} & \\
\hline & & Voltage-watt & & \\
\hline & & Dynamic current support & & \\
\hline & & Smooth frequency deviations & & \\
\hline \multirow{8}{*}{ Non-Autonomous } & \multirow{8}{*}{$\begin{array}{l}\text { - Communications and } \\
\text { control infrastructure } \\
\text { required } \\
\text { - Direct control of } \\
\text { inverter behavior } \\
\text { - Control from remote } \\
\text { operator commands or } \\
\text { feedback, based on } \\
\text { conditions at point of } \\
\text { common coupling }\end{array}$} & Command DER to connect/disconnect & \multirow{8}{*}{$\begin{array}{l}\text { SIWG } \\
\text { Phase } 3\end{array}$} & \\
\hline & & Limit real power & & \\
\hline & & Set real power & & \\
\hline & & Provide black-start capability & & \\
\hline & & Respond to real power pricing signals & & \\
\hline & & Participate in automatic generator control (AGC) & & \\
\hline & & Provide spinning reserves or bid into market & & \\
\hline & & $\begin{array}{l}\text { Update static set points for autonomous functions } \\
\text { (fixed power factor, volt-var curves, voltage ride- } \\
\text { through, frequency ride-through) }\end{array}$ & & \\
\hline
\end{tabular}




\section{Distributed Generation Interconnection Collaborative}
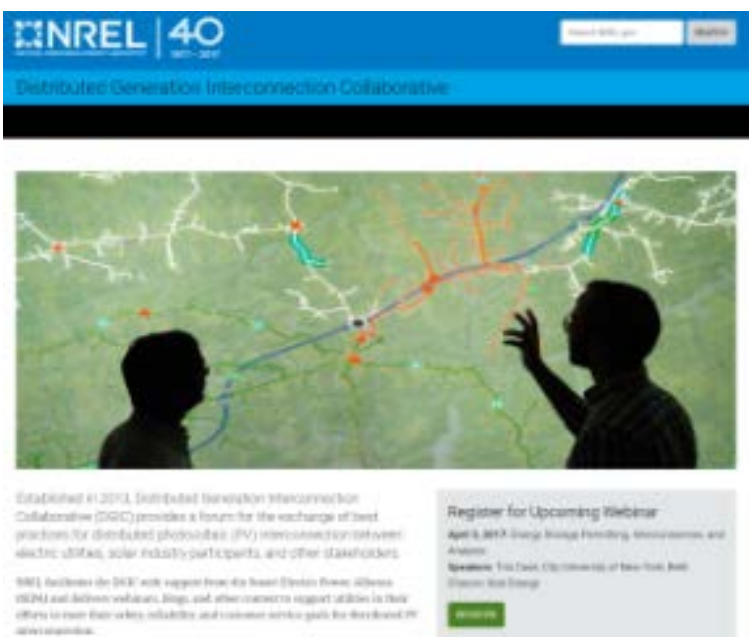

Utilities

Southern California Edison

Pacific Gas and Electric (PG\&E) (x2)

San Diego Gas and Electric (SDG\&E)

Sacramento Municipal Utility District (SMUD)

Hawaiian Electric Companies (HECO) (x2)

Arizona Public Service (APS) (x2)

Salt River Project (SRP)

Tucson Electric Power (TEP)

Xcel Energy

PEPCO

Con Edison

National Grid

Pasadena Water and Power

Fremont (NE) Dept of Utilities
- Funded by DOE SunShot Initiative

- Peer exchange on interconnection

- 17 webinars produced to date

- Next webinar: May 18, 2017 on interconnection for small utilities

$\begin{array}{ll}\text { Research Organizations } & \text { Utility Associations } \\ \text { RMI } & \text { NRECA } \\ \text { EPRI } & \text { WAPA } \\ \text { SEPA }(x 2) & \\ \text { City Univ. of New York (CUNY) } & \begin{array}{l}\text { Other Presenters } \\ \text { Borrego Solar (x2) } \\ \text { National Laboratories }\end{array} \\ \begin{array}{l}\text { NREL (x2) } \\ \text { Sandia }\end{array} & \text { ERCOT }\end{array}$

www.nrel.gov/dgic/ 


\section{Thank You!}

Emerson Reiter emerson.reiter@nrel.gov www.nrel.gov/dgic/

\section{www.nrel.gov}

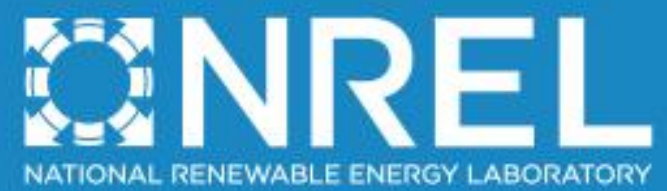

